\title{
Controlled coupling of counterpropagating whispering-gallery modes by a single Rayleigh scatterer: a classical problem in a quantum optical light
}

\author{
A. Mazzei, ${ }^{1}$ S. Götzinger, ${ }^{2}$ L. de S. Menezes,${ }^{3}$ G. Zumofen, ${ }^{2}$ O. Benson,, , 田 and V. Sandoghdar ${ }^{2, \oplus}$ \\ ${ }^{1}$ Nano-Optics, Humboldt University, Hausvogteiplatz 5-7, D-10117 Berlin, Germany \\ ${ }^{2}$ Laboratory of Physical Chemistry, ETH Zurich, CH-8093 Zurich, Switzerland \\ ${ }^{3}$ Departamento de Física, Universidade Federal de Pernambuco, 50670-901 Recife-PE, Brazil
}

\begin{abstract}
We present experiments where a single subwavelength scatterer is used to examine and control the back-scattering induced coupling between counterpropagating high-Q modes of a microsphere resonator. Our measurements reveal the standing wave character of the resulting symmetric and antisymmetric eigenmodes, their unbalanced intensity distributions, and the coherent nature of their coupling. We discuss our findings and the underlying classical physics in the framework common to quantum optics and provide a particularly intuitive explanation of the central processes.
\end{abstract}

The radiative properties of atoms can be strongly modified by coupling them to resonators [1]. A historical corner stone of this field of research, known as Cavity Quantum Electrodynamics (CQED), was set in 1946 by E. M. Purcell who proposed that the radiation rate of an oscillating dipole at wavelength $\lambda$ can be enhanced by a factor $F=3 Q \lambda^{3} / 4 \pi^{2} V_{m}$ in a resonant cavity of quality factor $Q$ and mode volume $V_{m}$ [1]. This socalled Purcell effect holds in the dissipative weak coupling regime where the cavity finesse is small so that the atomic radiation remains dominated by its coupling to the bath of the electromagnetic modes. In the strong coupling regime, coherent exchange of energy between the atom and the resonator causes the atomic resonance to lose its identity and to become replaced by a doublet. These phenomena have been studied for more than three decades [2, 3, 4, 5] although the in-situ manipulation of a single emitter in a single mode of a high-Q microresonator remains a challenge [4, 5]. In this Letter, we consider the controlled coupling of a classical nano-object to a high-finesse whispering-gallery mode (WGM) microresonator. We discuss both theoretically and experimentally the resulting coherent coupling between two degenerate counterpropagating WGMs and the modification of the Rayleigh scattering rate. Our findings show that the concepts of the strong and weak coupling play a central role even in this fully classical system.
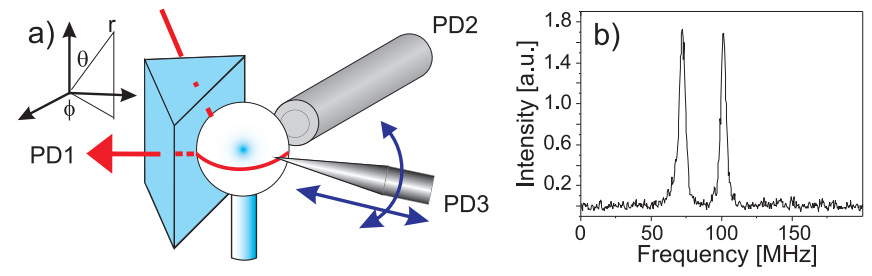

*Electronic address: oliver.benson@physik.hu-berlin.de

${ }^{\dagger}$ Electronic address: vahid.sandoghdar@ethz.ch
FIG. 1: a) Whispering gallery modes of a microsphere are excited via a prism. A glass fiber tip can be positioned in $(r, \theta, \phi)$ close to the sphere surface. The resonator spectrum can be recorded on PD1 in transmission, on PD2 from global scattering out of the sphere via a multimode fiber, and on PD3 through the fiber tip. b) An example of the doublet spectrum recorded on PD2.

The resonators in our work consist of microspheres melted at the end of silica fibers [6]. Such spheres support very high-Q WGMs and have been studied by several groups [7, 8, 9]. About ten years ago, it was discovered that the high-Q resonances of these cavities are often composed of doublets [10]. Such a mode splitting has been since discussed in conjunction with various WGM resonators 8, 9, 11, 12, 13]. It turns out that mode splitting has been observed in other ring resonators and has been explained as the result of the coupling between the electric fields $E_{c}$ and $E_{c c}$ of the degenerate clockwise (c) and counter clockwise (cc) modes via back scattering. The new superpositions states $(+)$ and $(-)$ are described by

$$
E_{+}=a E_{c}+b E_{c c} ; E_{-}=a E_{c}-b E_{c c} .
$$

Here $a$ and $b$ are complex coefficients. In the simplest case, the coupling between $E_{c}$ and $E_{c c}$ can be caused by a reflector [14, 15]. In the case of WGM resonators, however, it has been suggested that backscattering from a distribution of residual subwavelength inhomogeneities in the glass matrix or on its surface is the source of this coupling [8, 9, 10]. The orders of magnitude of the doublet splitting can be correctly estimated from classical electrodynamic considerations following this hypothesis [8, 9, 10, 12, 13, 16]. Nevertheless, the direct link between the spectral features of a doublet and the nanoscopic details of the backscattering sources has not been demonstrated experimentally, and a proper treatment of the losses inflicted by the scatterers is missing in the literature. In fact, an intuitively perplexing and interesting question arises in this context: given that the radiation of a subwavelength scatterer is nearly isotropic and that the angle subtended by a typical cavity mode is merely about $10^{-4} \mathrm{rad}$ [17], how could the rate of scat- 
tering back into a cavity mode dominate the rate of scattering out of the resonator to ensure the population of $E_{+}$and $E_{-}$?

The schematics of our experimental arrangement is shown in Fig. 17. A narrow-band diode laser $(\lambda=$ $670 \mathrm{~nm}$, linewidth $<300 \mathrm{kHz}$, tuning range $\approx 60 \mathrm{GHz}$ ) was used to excite the WGMs via a prism. Photodetector PD1 was used to record spectra in transmission through the prism whereas PD2 captured the light globally scattered out of the microsphere into a multimode fiber. Figure 10 displays a typical doublet with a splitting of $29 \mathrm{MHz}$ and $Q \simeq 8 \times 10^{7}$ recorded on PD2. The peaks represent the intensities $\left|E_{+}\right|^{2}$ and $\left|E_{-}\right|^{2}$ of the symmetric and antisymmetric eigenmodes. Following the procedure described in Ref. [18], we applied scanning near-field optical microscopy (SNOM) to map the spatial intensity distribution of the WGMs on PD3 and to identify the fundamental mode of the resonator, which exhibits a single intensity maximum in the $r$ and $\theta$ directions. In our previous works, we have shown that an uncoated glass tip might broaden and shift cavity modes depending on their $Q$ and on the tip size [19]. Here we demonstrate that a subwavelength tip can modify or induce the coupling between the degenerate $\mathrm{c}$ and $\mathrm{cc}$ microsphere modes.

Equation (1) implies that the interference between the $\mathrm{c}$ and cc running modes should give rise to sine and cosine standing waves along the equator. The locations of the nodes and antinodes of $E_{+}$and $E_{-}$are automatically established by the random distribution of a large number of inhomogeneities in the silica sphere [9, 10]. To visualize this effect, we have scanned a sharp fiber tip along the equator (i.e. in the $\phi$ direction) of an already split fundamental WGM and have recorded spectra at each point (note that the radial coordinate of the tip is kept constant using a shear-force feedback [18]). Figure [2a shows that the intensities of the two peaks of a doublet undergo out of phase periodic modulations as a function of the tip location. A slight slope of the middle base line is attributed to a drift in the shear-force tip-sphere stabilization. At location (i), the tip is positioned in the node of the symmetric mode and the antinode of the antisymmetric mode. Thus, it induces loss in the $E_{+}$mode while it leaves $E_{-}$nearly unaffected. Position (iii) shows the opposite counterpart of (i) whereas at position (ii) both modes are affected equally strongly.

As shown in Fig. 2b, the three spectra reveal that in addition to a change in the intensity balance of the doublets, their splittings are also modified. Figure $2 \mathrm{~d}$ displays the modulation of the splitting about its initial value of $24 \mathrm{MHz}$ shown by the dotted line. Interestingly, we find that the tip can not only increase the mode splitting, but it can also decrease it. This is due to a destructive interference between the field scattered by the tip and the field scattered by the inhomogeneities in the microsphere that gave rise to the initial splitting. Figures $2 \mathrm{r}$ and $\mathrm{f}$ provide further data on the increase and decrease of the mode splitting as the tip was scanned in the $\theta$ direction for two different $\phi$ positions spaced by half of the
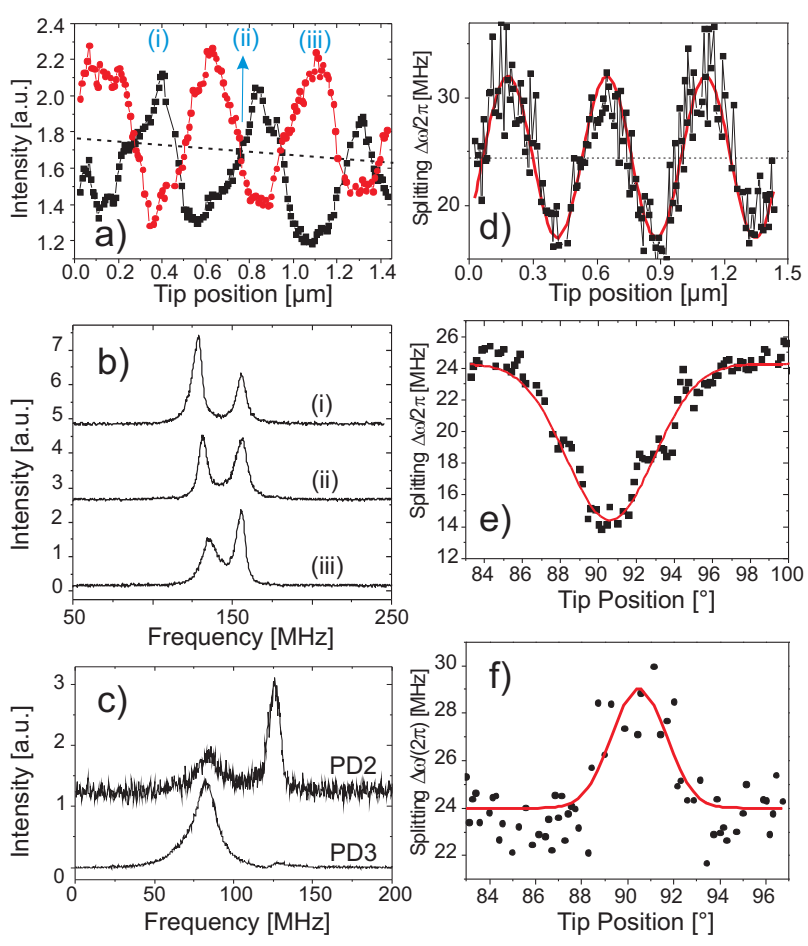

FIG. 2: a) The intensities of the two peaks in a doublet as a function of the tip position along the equator. The dotted line marks the slight intensity drift in the detection. b) Spectra recorded at positions (i-iii) in Fig. (a). The spectra are displaced vertically for clarity. c) Simultaneously measured spectra on PD2 and PD3. d) The recorded splitting corresponding to the data in Fig. (a). The solid curve shows a sinusoidal fit. e) and $\mathrm{f}$ ) The variations of the mode splitting as a function of the tip in the $\theta$ direction for positions (i) and (iii), respectively. The solid curves display fits according to the spatial mode function of the fundamental WGM.

interference period along the equator. Finally, Fig. 2 $2 \mathrm{c}$ plots the resonance spectra recorded simultaneously on PD2 and PD3, i.e. via global scattering from the sphere and via the fiber tip. The different lineshapes on the two channels might seem unexpected at first. However, this effect shows that if the tip is placed in an antinode of $E_{-}$ or $E_{+}$, it efficiently extracts photons out of that mode, leading to a larger signal in the fiber tip and thus a lower intensity in the cavity mode. On the contrary, the mode that is less perturbed is stronger in the resonator and is nearly uncoupled to the fiber tip. It is evident that the mode that is coupled to the tip has experienced an additional broadening.

We now show that the radiation properties of a subwavelength object such as its scattering rate are modified much in the same manner as those of the spontaneous emission of an atom. Our guiding thought is that many central features of CQED, including the modification of the mode density in a resonator, can be traced to the spatial character of the modes and should be thus shared by classical cavity electrodynamics. We first present a simple treatment of the free-space Rayleigh scattering using 
a semi-quantum electrodynamic (semi-QED) approach, where the material scatterer is treated classically while the field is quantized. Then we will discuss the modification of the scattering rate when the scatterer is coupled to a resonator.

Let us assume that a freely propagating photon is incident on a subwavelength spherical scatterer of radius $a$ and refractive index $n$. We take the photon to be in a mode $\widehat{E}_{k}$ with volume $V_{k}$, frequency $\omega_{k}$ and a linear polarization along the unit vector $\epsilon_{k}$ such that $\widehat{\mathbf{E}}_{k}=\mathcal{E}_{k} \epsilon_{k}\left(\widehat{a}_{k}^{\dagger}+\widehat{a}_{k}\right)$ where $\widehat{a}_{k}^{\dagger}$ and $\widehat{a}_{k}$ are the usual creation and annihilation operators and $\mathcal{E}_{k}=\sqrt{\hbar \omega_{k} / 2 \epsilon_{0} V_{k}}$. In the limit where the scatterer is considerably smaller than $\lambda$, it can be described by a dipolar polarizability $\alpha$ [20] so that the induced dipole moment operator reads $\widehat{\mathbf{p}}_{k}=\varepsilon_{0} \alpha \mathcal{E}_{k}\left(\widehat{a}_{k}^{\dagger}+\widehat{a}_{k}\right) \epsilon_{k}$. Thus the interaction energy between this dipole moment and another mode $\widehat{E}_{j}$ becomes,

$$
\widehat{\mathcal{V}}_{k, j}=-\widehat{\mathbf{p}}_{k} \cdot \widehat{\mathbf{E}}_{j}=\hbar g_{k j}\left(\widehat{a}_{k}^{\dagger} \widehat{a}_{j}+\widehat{a}_{j}^{\dagger} \widehat{a}_{k}\right)
$$

if we neglect the terms that do not conserve photon numbers. Here we have taken $g_{k j}=-\alpha \sqrt{\omega_{k} \omega_{j}} \mid \epsilon_{j}^{*}$. $\epsilon_{k} \mid / 2 \sqrt{V_{k} V_{v a c}}$ and have set $V_{j}=V_{v a c}$ for all vacuum modes $j$ into which the incident beam is scattered. The system Hamiltonian becomes [21]

$$
\widehat{H}=\hbar \omega_{k} \widehat{a}_{k}^{\dagger} \widehat{a}_{k}+\sum_{j} \hbar \omega_{j} \widehat{a}_{j}^{\dagger} \widehat{a}_{j}+\sum_{j} \hbar g_{k j}\left(\widehat{a}_{k}^{\dagger} \widehat{a}_{j}+\widehat{a}_{j}^{\dagger} \widehat{a}_{k}\right),
$$

leading to the Heisenberg equation of motion

$$
i \dot{\widehat{a}}_{k}=\omega_{k} \widehat{a}_{k}+\sum_{j} g_{k j} \widehat{a}_{j}
$$

The last term in Eq. (44) signifies the scattering of the incident field into all vacuum modes. Following the Weisskopf-Wigner formalism [22], we find the rate

$$
\Gamma_{R}=\frac{2 \omega_{k}^{2} V_{v a c}}{3 \pi c^{3}} g_{R}^{2}=\frac{\alpha^{2} \omega_{k}^{2}}{6 \pi c^{3} V_{k}}
$$

for this scattering event 21]. Here we have restricted ourselves to $\omega_{k}=\omega_{j}$ for elastic scattering and have used the notation $g_{R}=-\alpha \omega_{k} / 2 \sqrt{V_{k} V_{v a c}}$. Now we can calculate the Rayleigh scattering cross section $\sigma_{R}$ [20] by considering the total power radiated by the scatterer according to $I_{\text {inc }} \sigma_{R}=\hbar \omega_{k} \Gamma_{R}$. Given that $I_{\text {inc }}=\hbar \omega_{k} c / V_{k}$ and $\alpha=4 \pi a^{3}\left|\frac{n^{2}-1}{n^{2}+2}\right|$, one obtains the well-known relation

$$
\sigma_{R}=\frac{8 \pi k^{4} a^{6}}{3}\left|\frac{n^{2}-1}{n^{2}+2}\right|^{2} .
$$

We note that a rigorous quantum optical treatment of scattering is not frequently discussed in the literature 23] and goes beyond the scope of this paper. However, the fact that $\sigma_{R}$ can be derived via the Weisskopf-Wigner formalism using quantized fields provides a robust support for the intuitive expectation that a modification of the mode density, for example in a resonator or in front of a mirror, could also lead to a change in the Rayleigh scattering rate. The corresponding Purcell effect offers a physical explanation for the question posed earlier. The rate with which energy is transferred from $E_{c}$ to $E_{c c}$ is enhanced by $F$ and is given by $\eta F$ where $\eta$ is the geometric factor determining the fraction of the solid angle subtended by the mode. Therefore, a fundamental WGM with $V_{m} \simeq 130 \mu m^{3}$ (sphere diameter $30 \mu \mathrm{m}$ ) and $Q=10^{8}$ yields $F \sim 10^{4}$, compensating for the very small geometric acceptance of the order of $10^{-4}$. The influence of the Purcell factor $F$ also explains why reducing the cavity $Q$ results in the disappearance of light in the counterpropagating mode as reported previously [10].
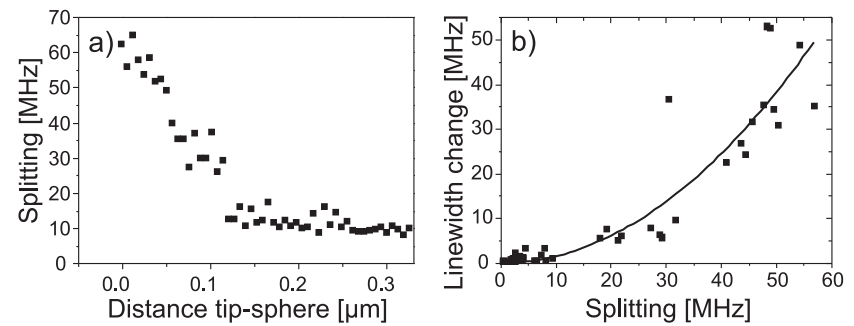

FIG. 3: a) The mode splitting measured as a function of sphere-tip separation in the $r$ direction. b) Plot of the measured splitting versus the additional tip-induced broadening. The solid curve displays the fit to a quadratic function.

Having shown that the density of states plays a central role in the description of Rayleigh scattering, next we consider the coupling of two counterpropagating cavity modes $E_{c}$ and $E_{c c}$ via a single Rayleigh scatterer. The details of the calculations are provided in the supplementary materials of this paper [21]. Returning to a classical notation, we find

$$
\begin{aligned}
& \dot{\tilde{E}}_{-}=\left(-i \Delta-\gamma_{0}\right) \tilde{E}_{-}+\kappa_{0} \\
& \tilde{\tilde{E}}_{+}=\left(-i \Delta+2 i g-\gamma_{0}-\Gamma\right) \tilde{E}_{+}+\kappa_{0} .
\end{aligned}
$$

Here we have defined $\widetilde{E}=e^{i \omega t} E, \Delta=\omega-\omega_{c}$ shows the detuning of the laser frequency $\omega, \kappa=\kappa_{0} e^{i \omega t}$ is the mode excitation rate, and $2 \gamma_{0}$ denotes the unperturbed cavity linewidth. When dealing with Rayleigh scattering out of a cavity, we have to take into account the spatial variation $f(\mathbf{r})$ of $E_{c}$ and $E_{c c}$ in the resonator mode. Going back to the definition of $g_{k j}$ and noting that $V_{j}=V_{k}$ is the WGM volume $V_{m}$ of the two modes, we thus obtain 21]

$$
\begin{aligned}
& 2 g=-\alpha f^{2}(\mathbf{r}) \omega_{c} / V_{m} \\
& \Gamma=\alpha^{2} f^{2}(\mathbf{r}) \omega_{c}^{4} / 6 \pi c^{3} V_{m}
\end{aligned}
$$

for the mode splitting and broadening, respectively.

A close scrutiny of Eq. (7) shows that if $|2 g|$ is sufficiently large to overcome $\gamma_{0}$ and if $2\left|\frac{n^{2}-1}{n^{2}+2}\right|<\left(\frac{\lambda}{2 \pi a}\right)^{3}$ to assure that $|2 g|>\Gamma$, a mode splitting is resolved. This is similar to the case of the strong coupling in CQED where the coherent exchange of energy between the cavity mode 
and an atom leads to a mode splitting if the coupling coefficient $|g|$ becomes larger than the cavity linewidth. As in CQED, the mode splitting $|2 g|$ grows with decreasing $V_{m}$ and increasing $\omega$, but the atomic dipole strength is replaced here by $\alpha$, and the splitting is asymmetric. The antisymmetric mode remains unperturbed (see Eq. (7)) whereas the symmetric mode undergoes an additional broadening given by $\Gamma$. This is a consequence of the fact that the phases of the new eigenmodes are self-adjusted so that the local scatterer is placed in a node (antinode) of the antisymmetric (symmetric) mode. This unbalanced splitting has been recently also observed for the coupling of a far-detuned cold atom ensemble to a high- $Q$ ring cavity [3]. Indeed, in that case several million atoms detuned from their transition resonances also behave essentially as a large dielectric object.

Equations (8) predict a linear dependence between the tip-induced splitting and line broadening if $f(\mathbf{r})$ is varied. However, due to the finite spatial extent of the tip and the WGMs, $\alpha$ effectively grows as the tip enters the mode. This position dependence leads then to a quadratic relationship between the tip-induced broadening and splitting. To investigate this effect, we have moved the tip in the radial direction and have recorded spectra at each location. Figure $3 \mathrm{~B}$ shows that as expected, the mode splitting becomes larger when the tip enters the evanescent field of the microsphere. Figure $3 \mathrm{~b}$ plots the tipinduced splitting versus the increase in the linewidth of $E_{+}$, confirming their quadratic dependence.

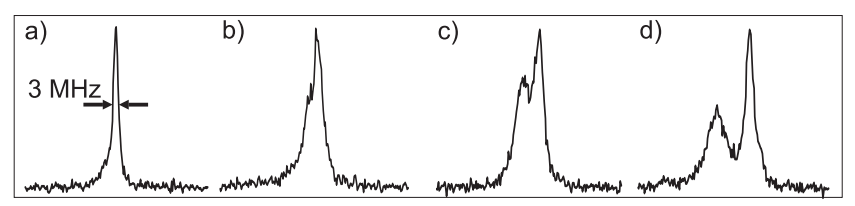

FIG. 4: a-d) WGM spectra recorded at different $\theta$ values of the tip location. At position a) the tip is nearly outside the spatial profile of the mode whereas at position d) it is in the mode maximum, i.e. the sphere equator.
To realize an ideal scenario for studying the interaction of a single well-defined scatterer with the fundamental mode of a microsphere, we have searched for spheres in which no mode splitting was observable in the beginning and it was created only when the tip was introduced. Figure 4 presents four spectra of a resonance as the tip was scanned in the $\theta$-direction from outside the mode (a) to the maximum of the mode at the equator (d) at constant separation from the sphere surface. The $E_{+}$ mode is shifted in frequency by $13 \mathrm{MHz}$ and broadened by about $6 \mathrm{MHz}$. Given that $|2 g| / \Gamma=3 \lambda^{3} / 4 \pi^{2} \alpha$ according to Eqs. (8), the observed ratio of the splitting to broadening implies a radius of $a \sim 140 \mathrm{~nm}$ for a spherical Rayleigh scatterer. This is in very good quantitative agreement with the experimental parameters, considering a typical value of $50-100 \mathrm{~nm}$ for the radii of curvature of SNOM tips and accounting for the overlap of the conical tip taper with the evanescent part of the mode.

In conclusion, we have considered the phenomenon of Rayleigh scattering both in free space and in the presence of a resonator. By using a semi-QED approach, we have pointed out the roles of the modification of the density of states and of the Purcell effect in classical scattering. Our results demonstrate that although the introduction of a scatterer into a high-finesse resonator might be commonly thought to introduce losses, it can mediate a coherent coupling of the resonator modes and cause their consequent normal mode splitting.

\section{Acknowledgments}

We are grateful for financial support from the Deutsche Forschungsgemeinschaft SP1113, the Swiss National Foundation (ETH group), NaFöG, Berlin (A. M.) and the Alexander von Humboldt Stiftung (L. de S. M.).
[1] P. R. Berman, Cavity Quantum Electrodynamics (Academic Press, 1994).

[2] P. Domokos, M. Gangl, and H. Ritsch, Opt. Commun. 185, 115 (2000).

[3] J. Klinner, M. Lindholdt, B. Nagorny, and A. Hemmerich, Phys. Rev. Lett. 96, 023002 (2006).

[4] T. Aoki, et al., Nature 443, 671 (2006).

[5] K. Hennessy, et al., Nature 445, 896 (2007).

[6] V. B. Braginsky, M. L. Gorodetsky, and V. S. Ilchenko, Phys. Lett. A 137, 393 (1989).

[7] L. Collot, V. Lefevre-Seguin, M. Brune, J. Raimond, and S. Haroche, Eur. Phys. Lett. 23, 327 (1993).

[8] T. J. Kippenberg, S. M. Spillane, and K. J. Vahala, Opt. Lett. 27, 1669 (2002).

[9] M. L. Gorodetsky, A. D. Pryamikov, and V. S. Ilchenko, J. Opt. Soc. Am. B 17, 1051 (2000).
[10] D. S. Weiss, et al., Opt. Lett. 20, 1835 (1995).

[11] J. Krupka, P. Blondy, D. Cros, P. Guillon, and R. G. Geyer, IEEE Trans. Micro. Theo. Tech. 44, 1097 (1996).

[12] M. Borselli, T. J. Johnson, and O. Painter, Opt. Exp. 13, 1515 (2005).

[13] K. Srinivasan and O. Painter, Phys. Rev. A 55, 023814 (2007).

[14] R. J. C. Spreeuw and J. P. Woerdman, Physica B 75, 96 (1991).

[15] A. Venugopalan, D. Kumar, and R. Ghosh, Pramana J. Phys. 40, 107 (1993).

[16] P.-Y. Bourgeois and V. Giordano, IEEE Trans. Microw. Theo. Tech. 53, 3185 (2005).

[17] S. Götzinger, et al., Nano Lett. 6, 1151 (2006).

[18] A. Mazzei, S. Götzinger, L. de S. Menezes, V. Sandoghdar, and O. Benson, Opt. Comm. 250, 428 (2005). 
[19] S. Götzinger, O. Benson, and V. Sandoghdar, Opt. Lett. 27, 80 (2002).

[20] D. Jackson, Classical Electrodynamics (Wiley and Sons, 1999).

[21] The EPAPS Supplementary material presents the details of the calculations leading to equations (5) and (7).
[22] P. W. Milonni, The Quantum Vacuum (Academic Press, New York, 1994).

[23] B. J. Dalton, S. M. Barnette, and P. L. Knight, J. Mod. Opt. 46, 1107 (1999). 